\title{
Reconstruction of the gastrointestinal tract after total gastrectomy
}

\begin{abstract}
Currently, there are more than 70 types of restoration of gastrointestinal tract (GIT) continuity. Reconstruction of the digestive tract is an important part of surgery on par with lymph node dissection and it must have the following characteristics: gradual emptying into the small intestine, prevention of reflux esophagitis and dumping syndrome, small reservoir size, and reconstructive operative procedures should be relatively uncomplicated and not time-consuming. The technique of GIT reconstruction is the determining factor of postoperative quality of life for the patients. According to the Japanese guidelines, there're two types of reconstruction: with preservation of the duodenal passage and without it. The most known techniques in the duodenal passage exclusion group are Roux-en-Y reconstruction and Roux-en-Y reconstruction with pouch formation. In the duodenal passage preservation group interposition of the jejunum with and without pouch formation are the most common. Currently, Roux-en-Y reconstruction is the preferred technique. The use of Roux-en-Y reconstruction with a pouch to recover the initial anatomical structure significantly decreases the incidence of dumping syndrome and doesn't increase the number of postoperative complications or mortality, operative time, hospitalization time, and significantly increases patients' quality of life. For laparoscopic total gastrectomy, it was shown that intracorporeal anastomosis has a better cosmetic appearance, it's safer, technically feasible, less invasive compared to the extracorporeal anastomosis. Reconstruction using the OrVil device should be used in patients with tumors of the cardia or lower third of the esophagus because anastomosis can be formed high in the mediastinum which increases the probability of negative proximal resection margin after gastrectomy.
\end{abstract}

Keywords: gastrectomy, intracorporeal anastomosis, anastomosis, gastrointestinal tract, gastric cancer
Volume 10 Issue 4 - 2019

\author{
Pavlov Rostislav,' Ishenko Roman, ${ }^{2}$ IIlya \\ Shpektorovskyy,3 Pavlenko Andrew,' Sakharov \\ Alex' \\ IDepartment of oncology, Saint-Petersburg Clinic of Advance \\ medical technology, Russia \\ 2 Department of surgery, Federal Scientific Clinical Center, Russia \\ 3State Establishment "Dnipropetrovsk State Medical Academy of \\ Health Ministry of Ukraine, Ukraine
}

Correspondence: Rostislav Pavlov, Saint-Petersburg University Clinic of Advanced Medical Technologies, Oncology department, Saint Petersburg, Russia, Tel +79522182188,

Email onco_spb@mail.ru

Received: June 27, 2019 | Published: August 01, 2019
Abbreviations: GI, gastric cancer; RAO, robot-assisted operations; NDPP, non-duodenal passage preservation; DPP, nonduodenal passage preservation; $\mathrm{Hb}$, hemoglobin, SI, serum iron; DT, double tract

\section{Introduction}

Adenocarcinoma of the stomach was the leading cause of cancer mortality in the world for the most part of the 20th century. Currently, gastric cancer is the 5th most common cancer after lung, breast, colorectal and prostate cancers. At the same time, gastric cancer is the 3 rd leading cause of cancer mortality in men and women worldwide despite significant improvements in diagnosis and treatment. The highest mortality rates are observed in Eastern Asia, the lowest in North America. Annually, 989,600 new cases are diagnosed, and 738,000 deaths are registered worldwide ( $10 \%$ of all cancer deaths). ${ }^{1}$

Complete removal of the stomach is a difficult operation both for the surgeon and the patient. There are a significant number of postoperative complications leading to unwelcome consequences for the patient. Gastrectomy is an operation causing an interruption of the physio-anatomical function of the gastrointestinal tract (GIT). Reconstruction of the gastrointestinal tract is an important part of the surgery, as well as lymph node dissection, and must satisfy the following criteria: gradual emptying into the small intestine, prevention of reflux esophagitis and dumping syndrome, small reservoir size, and reconstructive operative procedures should be relatively uncomplicated and not time-consuming. ${ }^{2}$ The method of GIT reconstruction is the determining factor of the patient's quality of life. It includes general and specific complaints, social and psychoemotional factors. Measurement of postoperative quality of life (PAL) is made possible by the development of various scales (Visick, Karnofsky, Spitzer's index, QLQ-C67, et al.).

Currently, more than 70 types of GIT patency reconstruction had been tested. However, the surgical community still disagrees on the best reconstruction method. In this literature review, we plan to consider different types of reconstructions for open, laparoscopic and robot-assisted operations (RAO), analyze the results of studies dedicated to PAL, postoperative complications, reflux esophagitis, dumping syndrome, anastomosis failure, reconstruction operative time for different GIT reconstruction techniques. The studies were selected from the PubMed, Cochrane Library, Up-to-date databases.

The first gastrectomy in the world was performed by Conner in 1884. ${ }^{3}$ The first successful gastrectomy was performed by Schlatter in 1897. ${ }^{4}$ In his monograph on gastric cancer published in 1966, Zoltan Sabolch described 58 different types of reconstruction after gastrectomy. The high number of different types of reconstruction points at the absence of an optimal solution for this problem. According to the Japanese guidelines, ${ }^{5}$ there're two types of reconstruction: with preservation of the passage through the duodenum and without it. The most known in the group of surgeries with exclusion of the duodenal passage are Roux-en-Y procedure and Roux-en-Y reconstruction with formation of a pouch. In the group of duodenal passage preservation, interposition of the small intestine with and without formation of a pouch is the most common. 
The obvious benefit of preservation of the duodenum continuity is endoscopic accessibility of the duodenum and biliopancreatic duodenal zone. Henley ${ }^{6}$ first published the results of this reconstructive technique in 1953. Reconstruction of continuity between the esophagus and the duodenum with interposition of the jejunal loop appears to be a more physiological method; this type of reconstruction increases operative time by 25-40 minutes compared to Roux-en-Y reconstruction without an increase in postoperative mortality and postoperative complications but involves an additional anastomosis and increased bile reflux. None of the randomized clinical trials (RCTs) show concrete proof of the advantage of esophagoduodenal anastomosis (EDA) with interposition of the jejunal loop over Roux-en-Y reconstruction..$^{7-10}$ In an RCT involving 106 patients, interposition of the jejunum with a pouch was compared to Roux-en-Y reconstruction with a pouch. The authors didn't observe any significant differences in PAL (Visick score and Spitz's scale). ${ }^{11}$ Altogether, the majority of these trials wasn't able to demonstrate any significant benefits in terms of decreased risk of dumping syndrome, onset of esophagitis, or GIT improvement in patients. Therefore, preservation of the duodenal passage increases operative time without an increase in postoperative complications. Some interesting results were obtained in a meta-analysis. ${ }^{12}$ published in 2013. It included 9 randomized trials involving 642 patients, 325 of which underwent non-duodenal passage preservation (NDPP) and 317 underwent reconstruction with duodenal passage preservation (DPP). The meta-analysis had shown that preservation of the duodenal passage didn't increase the rate of postoperative complications and mortality but significantly increased operative time $(p<0.0001)$. No significant differences between the patients' quality of life in the two groups in the early and late postoperative period were observed. Seven RCT reported on the changes in body mass dynamics in various observational periods. Among them, three studies reported on body mass changes at 3 and 6months after the surgery. The meta-analysis had shown that the NDPP group exceeded the DPP group in terms of body mass after radical gastrectomy 3 and 6 months post-surgery. ${ }^{12}$ Descriptive analysis was used in the other five RCTs due to the absence of specific data. There were no significant differences between the two reconstructions 12 months after the surgery. Additionally, for more long-term observations, no significant differences in the results were detected for the whole follow-up period: for two, there or five years. DPP promoted improved levels of such serum nutritional markers as albumin, hemoglobin $(\mathrm{Hb})$, serum iron (SI). Apart from serum albumin level 6 months after the surgery, there were no significant differences between the two reconstruction techniques. The results of 3 RCTs had shown that DPP doesn't significantly reduce heartburn symptoms compared to NDPP. Moreover, there were no significant differences in the development of heartburn between the two groups after the surgery. The incidence of dumping syndrome was lower in the DPP group compared to the NDPP group 3, 6 and 24 months after the surgery. The longer follow-up duration showed no significant differences. Therefore, DPP didn't lead to an increased number of postoperative complications or mortality but increased working time of the surgical team. Additionally, the results demonstrated increased body mass and higher serum parameters $(\mathrm{Hb}, \mathrm{SI})$ in the early postoperative period in the DPP group. However, no significant differences in heartburn incidence were observed in the two groups after the surgery. For longer follow-up periods, there was no difference in dumping syndrome incidence. There were no significant differences in quality of life (Visick score and Spitz's scale) between the two groups which puts into question the advantage of DPP.
The group of duodenal passage preservation also includes double tract (DT) reconstruction introduced in 1965 by Kajitani and Sato. This procedure allows to perform esophagojejunal anastomosis (EJA) similar to Roux-en-Y reconstruction, and $20 \mathrm{~cm}$ distally from the EJA a duodenojejunal anastomosis is added..$^{13}$ In the period from 2012 to 2016, 110 patients with gastric adenocarcinoma diagnosis aged 35-74years were randomized in the trial. ${ }^{14}$ The objective was to compare two GIT reconstructions (Roux-en-Y and DT) after total gastrectomy, D2 lymph node dissection and omentectomy for treatment of gastric cancer. Reconstruction type was chosen randomly. This trial was designed as a stratified randomized trial with two groups in accordance with the reconstruction type. Fiftyone (51) patients underwent Roux-en-Y reconstruction, 59 underwent DT reconstruction. There was a significant difference between the two groups concerning body mass index (BMI) 12 months after the surgery $(p<0.001)$. Mean operative time for Roux-en-Y reconstruction was 193.41minutes, DT reconstruction -216.01 minutes $(p<0.001)$. Evaluation of PAL was performed 12 months after the surgery using the QLQ-C30 questionnaire. No significant differences between PAL in the two groups were observed $(p>0.05)$. The total number of EJA failures was six, and they were equally distributed between the two groups: $5.9 \%$ patients in the Roux-en-Y group and $5.1 \%$ patients in the DT group $(p>0.05)$. In 9 patients, EJA strictures were diagnosed 12 months post-surgery: in $7.84 \%$ of patients in the Roux-en-Y group and $8.47 \%$ patients in the DT group $(p>0.05)$. Considering the obtained data on the number of anastomosis failures, stricture formation, PAL, we can conclude that there's no significant advantage of DT reconstruction compared to Roux-en-Y.

Currently, the most common solution for GIT reconstruction after gastrectomy is Roux-en-Y reconstruction based on the Cezar Roux's concept used after partial gastric resection in $1892 .{ }^{15}$ Orr was the first to apply this technique after radical gastrectomy in $1947 .{ }^{16}$ In 1952 , Hunt published his technique for double suturing of the jejunum at the proximal end of the Roux-Y loop and creation of a reservoir by side-by-side suturing of a jejunojejunal anastomosis (JJA). ${ }^{17}$ The Hunt-Lawrence-Rodino anastomosis described in a 1988 review to this day remains the most known type of pouch. ${ }^{18}$ Theoretically, addition of a pouch recreates anatomical and physiological function of the stomach and, therefore increases stomach capacity, maintains body mass and preserves quality of life. Nineteen randomized studies involving 983 patients in total had evaluated the advantages of a pouch. In these trials, use of a pouch increased postoperative quality of life (Visick score) ${ }^{19}$ In their study involving 138 patients, Fein et al. ${ }^{20}$ demonstrated that in the first postoperative year, there were no advantages in terms of quality of life after Roux-en-Y reconstruction with a pouch but 30 months later a significant increase in quality of life compared to Roux-en-Y reconstruction without a pouch was observed. Weight loss was the same in the two groups. There were no differences in operative time, postoperative complications and mortality. The research team had made a very interesting conclusion. They stated that cancer advancement, instead of the type of reconstruction, determines PAL, and recommended not to perform reconstruction with a pouch in patients with advanced stage gastric cancer. In the QUOROM meta-analysis, patients after radical gastrectomy using Roux-Y reconstruction with or without pouch were followed up for 15 months. ${ }^{21}$ The results showed that formation of a reservoir didn't lead to a significant increase in complications (58/187 versus $63 / 200 ; p=0.71$ ) or mortality $(9 / 187$ versus $8 / 200 ; p=0.93)$. No significant differences were observed in operative time between the 
two groups (mean difference was 8 minutes; $p=0.38$ ) or the number of hospitalization days (mean difference was 1 day; $p=0.81$ ).

Data on dumping syndrome were analyzed for three time perimonths. A decrease in the incidence of dumping syndrome was observed in the pouch group 6 and months post-surgery (6/33 versus $12 / 29 ; p=0.003$ after 6 months and $6 / 58$ versus $15 / 51 ; \mathrm{p}=0.01$ after 12 months). Heartburn incidence was also lower in the Roux-en-Y pouch reconstruction group 1 year after the surgery ( $1 / 19$ versus $7 / 18$; $p=0.03$ ). Food intake was higher in the patients with a pouch compared to patients without a pouch with significant differences $12-15$ months after the surgery (food intake $\leq 50 \%$ from the preoperative level: $7 / 42$ versus $16 / 32 ; p=0.003)$. However, there were no differences in food intake between the groups after 5years. Patients with R0 resections (33), 5-year survival (36) and reconstruction with a pouch had shown a much better postoperative quality of life 12 months $(p=0.03)$ and 24 months after the surgery $(p=0.01)$. Therefore, this meta-analysis confirms that Roux-en-Y reconstruction with a pouch after radical gastrectomy significantly decreases the incidence of dumping syndrome, doesn't increase postoperative complications or mortality, operative time and hospitalization time and significantly increases patients' quality of life post-surgery.

Encouraged by the results, surgeons have developed various modifications of the Hunt-Lawrence-Rodino pouch: Ligidakis modification, J-pouch, aboral pouch. Multicenter prospective RCT CCG1101. ${ }^{22}$ was designed to test the hypothesis that the aboral pouch (AP) leads to favorable postoperative results. The patients were randomized into 2 groups. The first group underwent Rouxen-Y reconstruction (51 patients), the second group also received the aboral pouch (49 patients) after radical gastrectomy for gastric cancer. The study results showed no significant differences between the two groups in intraoperative blood loss, operative time, and number of hospitalization days, complications and hospital mortality. No APspecific complications were reported. The study sample wasn't large enough for identification of significant benefits of AP, only nausea and vomiting symptoms were significantly improved in the AP group ( $p=0.041) 1$ year post-surgery.

Chen et al. ${ }^{23}$ demonstrated the results of 60 patients who underwent total gastrectomy with J-pouch reconstruction (32 patients) or traditional Roux-en-Y reconstruction (28 patients). Quality of life was evaluated using the Visick score and Spitzer index. Three (3) months after the surgery, in the J-pouch group lower discomfort in the abdomen, appetite loss, and weight loss were observed compared to the Roux-en-Y group (3-month Spitzer index: $28.1 \pm 4.0$ versus $24.3 \pm 4.9 ; p=0.002)$. Similar results were observed at 6 and 12 months after the surgery (6-month Spitzer index: $31.3 \pm 4.0$ versus $26.3 \pm 4.8$, $p=0.000$; 12 -month Spitzer index: $32.7 \pm 3.4$ versus $27.8 \pm 4.8, p=0.000$ ). Similar results were obtained for the Visick score in the two groups. Differences in data were observed at two time points (3 and 6 months) which were identified by single factor analysis (after 3 months: $p=0.005$; after 6months: $p=0.028$ ). Dumping syndrome and reflux esophagitis were more common in the Roux-en-Y reconstruction group ( $p=0.021$ and $p=0.04$ ). In 2016, Zonca et al. ${ }^{24}$ in their RCT also demonstrated positive results for J-pouch reconstruction compared to Roux-en-Y reconstruction after total gastrectomy. Mean operative time was 226.7 minutes in the J-pouch group and 178.1 minutes in the Roux-en-Y group $(p<0.05)$. Patients didn't have anastomosis failure or bowel obstruction. Evaluation of quality of life was performed using the Eypasch questionnaire one year after the surgery. Quality of life score in the Roux-en-Y reconstruction group was $106 \pm 18.8$ (mean standard deviation). Patients with J-pouch reconstruction had the quality of life score of $122 \pm 22.5$. It was shown that the difference was significant ( $p<0.0016$, t-test). According to the obtained results, patients with J-pouch reconstruction showed significantly higher quality of life compared to patients with Roux-en-Y reconstruction. Therefore, the study results show that radical gastrectomy with J-pouch reconstruction is a feasible and safe procedure for treatment of gastric cancer. Clear improvement in PAL, dumping syndrome incidence, reflux esophagitis point to the superiority of this reconstruction methods compared to Roux-en-Y reconstruction. During the formation of a pouch, surgeons must consider the size of a pouch. A proximal pouch formed using the duodenum is a traditional variant and its size varies between 7 and $20 \mathrm{~cm}$. In their meta-analysis, Dong et al. ${ }^{25}$ had demonstrated that small pouches $(11 \mathrm{~cm})$ improve PAL by decreasing post-gastrectomy symptoms including heartburn, dysphagia, and vomiting.

In 1993 in Belgium, Azagra performed the first laparoscopic total gastrectomy for treatment of gastric cancer, and in 1999 Azafra et al. reported on 13 patients who underwent laparoscopic gastrectomy. They concluded that laparoscopy is a valid method of gastric cancer treatment and requires further study. ${ }^{26}$ In 2006, the same authors participated in a multicenter trial analyzing 130 patients with gastric adenocarcinoma and mean follow-up period of 49 months. The study showed that laparoscopic gastrectomy with any type of lymph node dissection and even as a palliative therapy is a safe procedure with acceptable mortality and morbidity results in patients with advanced gastric cancer. ${ }^{27}$ Intracorporeal anastomosis (IA) and extracorporeal anastomosis (EA) after laparoscopic total gastrectomy (LTG) have some differences. First of all, IA provides less tension applied to the anastomosis and allows avoiding injuries of the surrounding structures. Secondly, IA doesn't require additional cuts with a lesser impact on the working area. Thirdly, IA is performed under close visualizing control. These characteristics of IA lead to a smaller number of postoperative complications and promote faster recovery after the surgery. Zheng et al. ${ }^{28}$ in a meta-analysis had shown that IA is characterized by better appearance, lower blood loss and faster recovery of intestinal function. In $\mathrm{RCT}^{29}$ and meta-analysis ${ }^{30}$ of 2015, LTG with IA was reported to be safer and more technically feasible that LTG with EA.

Otsuka et al. ${ }^{31}$ state that Roux-en-Y reconstruction is one of the typical variants of LTG for treatment of gastric cancer. However, gastroduodenoscopy can be quite difficult after this procedure. Taking into account periodical endoscopic examinations of the duodenum is strongly recommended for post-surgery patients with concomitant disorders such as duodenal adenoma, familial polyposis or Crohn's disease. Therefore, reconstructions with preservation of the duodenal passage could be preferential for these patients. Kanaya et al. ${ }^{32}$ proposed using DT reconstruction after LTG because this method requires only a small modification of the Roux-en-Y reconstruction procedure. For this modification, a delta-shape intracorporeal anastomosis was used which was developed for B-1 reconstruction after distal gastrectomy. Advantages of DT reconstruction are that this technique is relatively easy to perform, preserves chyme passage through the duodenum, and doesn't require creation of a duodenal stump. Additionally, DT reconstruction allows to significantly simplifying endoscopic access to the duodenum after the surgery compared to Roux-en-Y reconstruction. ${ }^{33}$ In an effort to simplify the procedure of anastomosis creation, surgeons started to perform end- 
to-end delta-shape anastomosis after LTG. However, Zuiki et al. ${ }^{34}$ in their study demonstrated a high incidence of stenosis after LG with end-to-end delta-shape anastomosis. The size of circular stapler is an important factor, and previous studies showed that the use of $21 \mathrm{~mm}$ stapler is an independent risk factor and requires another choice of reconstruction in patients with small-diameter esophagus. Fukagawa et al. ${ }^{35}$ reported on the presence of stenoses in delta-shape EJA after total gastrectomy in $4.1 \%(60 / 1,478)$ patients in the National Cancer Center Japan. They concluded that the use of small diameter stapler $(21 \mathrm{~mm})$ is an important risk factor for anastomotic stenosis.

The high incidence of failures and strictures of EJA prevented the advancement of LTG use for traditional end-to-side anastomoses. Jeong et al. ${ }^{36}$ reported that EJA stricture incidence after LTG was $3.4 \%$, EJA failure incidence was $7.6 \%$. Zuiki et al. ${ }^{37}$ reported that a retrospective study had shown that the incidence of EJA strictures after LTG was $21 \%$, the failure rate was $1.9 \%$. To decrease the incidence of the aforementioned complications, Duan at al. ${ }^{38}$ developed a semiend-to-end (SETE) anastomosis for Roux-en-Y reconstruction after LTG. The study results demonstrated that mean recovery time in the SETE group was significantly lower than in the end-to-side group. Results of this study showed that the rate of complications associated with EJA in the SETE group was lower than in the end-to-side group, and the incidence of stricture formation was lower in the SETE group. In the SETE procedure, strictures could form with a low probability because anastomosis canal corresponded in size to the esophageal canal. The failure rate was lower in the SETE group but the difference was insignificant. For the SETE procedure, an anastomosis could be easily put in the esophageal opening without the jejunum which lowered the risk of failure. Additional RCTs are necessary for a better understanding of the necessity of SETE anastomosis.

In the last 5 years, several studies were published describing positive results of using the OrVil device which serves as the most common reconstruction method in laparoscopy. Wada et al. ${ }^{39}$ reported mean operative time of 249 minutes, blood loss of $182 \mathrm{ml}$ and anastomosis failure rate of $6 \%$ in 100 patients who underwent laparoscopic gastrectomy using the OrVil device. Zuiki et al. ${ }^{40}$ also reported that the rate of anastomosis failure was $1.9 \%$, stenosis incidence was $21.2 \%$ ( $75 \%$ with $21 \mathrm{~mm}$ anvil and $17.0 \%$ with $25 \mathrm{~mm}$ anvil) in 52 patients. Abdelmonim et al. ${ }^{41}$ believe that the OrVil device has significant advantages compared to traditional suturing methods for patients with oncological diseases. One of the main advantages is that anastomosis can be formed high in the mediastinum in patients with cardiac tumors without the necessity of thoracotomy. This was especially useful for patients with tumors of the cardia or lower third of the esophagus. In this study, 29 anastomoses (40.3\%) were performed laparoscopically in the lower part of the thorax, and 23 of them $(79 \%)$ didn't require thoracotomy. An additional advantage is that the use of the OrVil technique increases the probability of negative proximal resection margin after gastrectomy. Using the OrVil device, anastomosis can be created much higher than for manual anastomosis which complicates a surgeon's work in this area. This is confirmed by the fact that this study had only three positive proximal resection margins, and in all three cases resection was performed for palliative reasons. Positive resection margin rate for this technique varies between 0 and $12.5 \%$ and lower in different articles. Stricture rate was $9.7 \%$. In a single-center study of 117 patients ${ }^{41}$ who underwent radical LG using the OrVil device, mean operative time was 243 minutes, mean blood loss was $79 \mathrm{ml}$, EJA failure rate was $1.7 \%$ stenosis incidence was $1.7 \%$. In this study, the rate of EJA failure was lower in patients who underwent radical LG using OrVil than in patients who underwent open gastrectomy, though the difference wasn't significant. Additionally, intraoperative blood loss in radical LG was significantly lower than in the laparotomy group. This study had some limitations. Firstly, there were differences between the patients in the surgical groups. Patients who underwent open surgery had more advanced cancer. Secondly, the surgical procedures were performed in different time periods. Open surgery has been performed since the foundation of the institute while radical LG using the OrVil device has been performed since 2007. Therefore, different results associated with the procedure such as instruments, sutures, and drugs could affect the results. Additionally, there could be differences in the practical skills of the surgeons performing the procedures and preoperative protocols of patient management.

\section{Materials and methods}

Between 2016 and 2019 at the Saint-Petersburg University Clinic of Advanced Medical Technologies gastrectomy surgeries were performed on patients with advanced gastric cancer with two types of reconstruction: in Group 1 (39 patients) standard operation with stapling Roux-en-Y reconstruction was performed; in Group 2 (41 patients) operation with manual reconstruction and interposition of the jejunum was performed. Patient age varied between 35 and 74years. All patients underwent standard preoperative complex of examinations including endoscopic gastroduodenoscopy with biopsy, contrast-enhanced computed tomography of the three zones. We studied operative time, blood loss, postoperative complications, postoperative quality of life, and variations in body mass. Evaluation of the patients' quality of life was performed 1 month and 1year after the surgery.

\section{Results}

Analysis of the results showed that difference in operative time was more than 20minutes: in Group 1 (Roux-en-Y reconstruction) it was 195.33minutes, in Group 2 (manual interposition of the jejunum) it was 218.87 minutes $(p=0.05)$. Blood loss was 120 and $150 \mathrm{ml}$ in Group 1 and Group 2 respectively $(p=0.05)$. However, there were no significant differences in the rate of postoperative complications between the two groups. Dumping syndrome and reflux esophagitis were observed in 33 patients $(84.6 \%)$ in Group 1 and in 34 patients $(82.9 \%)$ in Group $2(p=0.05)$. The EORTC QLQ-STO 22 questionnaire was used to evaluate the patients' quality of life 1,3,6 months and 1 year after the surgery. Analysis of the questionnaire results prior to surgery showed that mean level of quality of life for the patients was $65.3 \pm 9.1$. One month after the surgery, positive dynamic in the patients' self-assessed condition was observed in both groups, and higher mean score of $76.4 \pm 2.2$ was observed in Group 1. In Group 2, mean score was $71.9 \pm 1.5(p=0.05)$. In both groups, the patients noted a decrease in such symptoms as pain, dysphagia, reflux symptoms, as well as improved emotional status compared to their status prior to the surgery. After 3 months, patients in both groups showed a significant increase in emotional scores compared to the preoperative values $(p=0.05)$. Six months after gastrectomy, the patients in Group 1 noted increased frequency of nausea and vomiting, diarrhea and constipation $(p=0.05)$. The patients in Group 2 had a higher increase in pain and diarrhea $(p=0.05)$. One year after gastrectomy, a second stage of evaluation of quality of life was performed. Mean scores in the two groups were close (66.3 and 65.7 in Group 1 and Group 2 respectively) $(p=0.05)$. The patient started to complain of 
reflux esophagitis, nausea and vomiting, diarrhea. The data showed that $22(56.4 \%)$ and $24(58.3 \%)$ patients in Group 1 and Group 2 respectively returned to the baseline performance status (ECOG-1) after gastrectomy $(p=0.05)$. The values of symptom scores per the EORTC QLQ-STO22 questionnaire are presented in Figure 1.

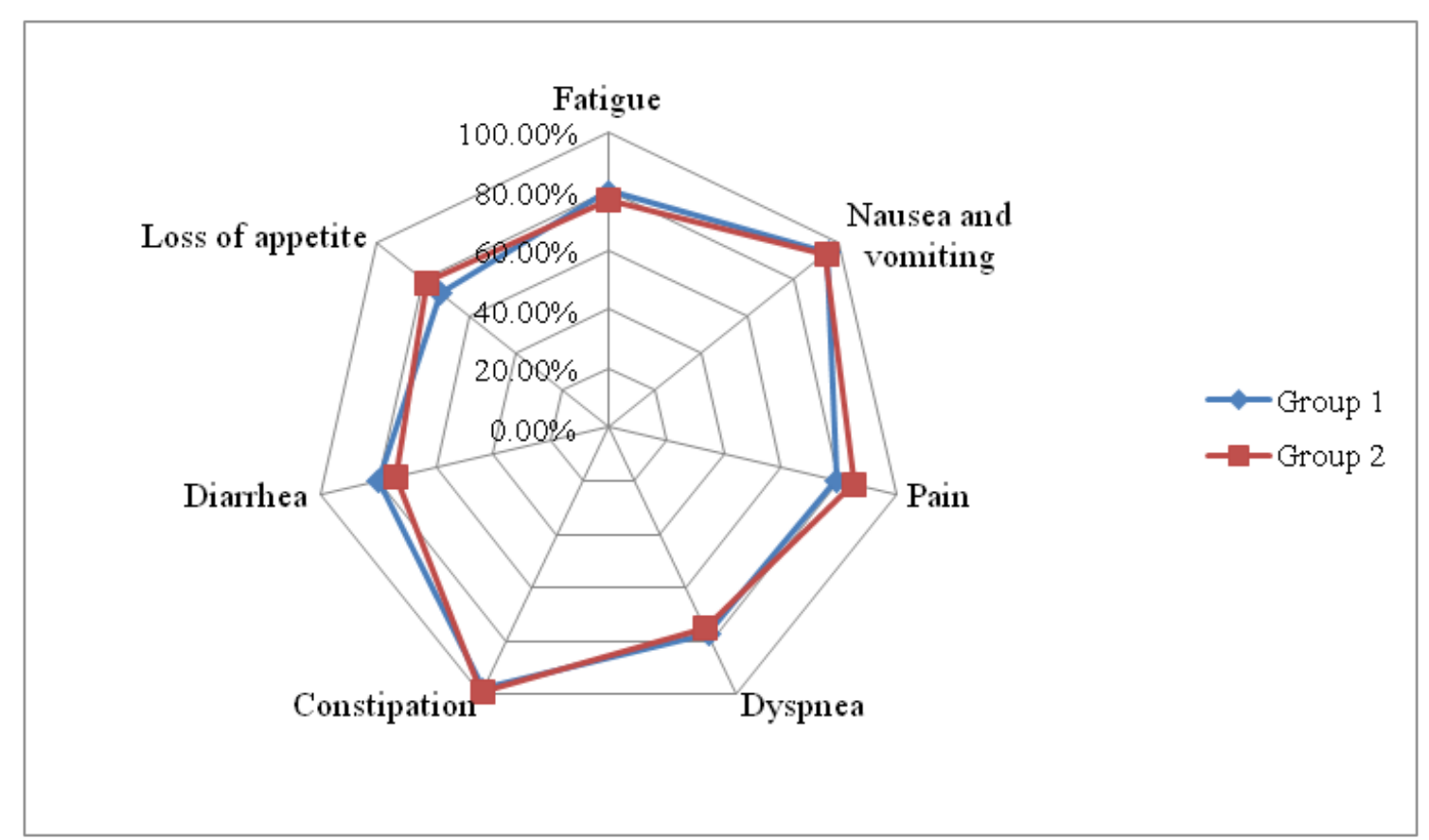

Figure I. Taking into account the absence of significant differences in postoperative complications and quality of life, we consider stapling Roux-en-Y reconstruction a more preferable and practical operation.

\section{Discussion}

Currently, surgeons have two types of anastomoses that can be performed in patients after total gastrectomy: GIT reconstruction with preservation (interposition of the jejunum) and without preservation (Roux-en-Y) of the duodenal passage. The majority of the studies failed to demonstrate any significant advantages in terms of decreased risk of dumping syndrome, reflux esophagitis or PAL improvement in patients who underwent interposition of the jejunum. Therefore, preservation of the duodenal passage increases operative time without a decrease in postoperative complications which puts into question its advantage compared to Roux-en-Y reconstruction..$^{7-12}$ In Europe, Roux-en-Y reconstruction is the preferred procedure. This procedure is simple and almost always possible: isoperistaltic loop of the jejunum is lifted and anastomosed with the distal part of the esophagus. A European surgical team in an attempt to recover the initial anatomical structure proposed using a pouch between the esophagus and the duodenum. Generally, the studies confirm that Roux-en-Y reconstruction with a pouch after radical gastrectomy significantly decreases the incidence of dumping syndrome, doesn't increase postoperative complications or mortality, operative time, hospitalization time and significantly increases patients' PAL. ${ }^{19-21}$ In the era of laparoscopic surgery, the problem of the best reconstruction technique after laparoscopic total gastrectomy has been widely discussed. Two meta-analyse ${ }^{28,30}$ and one RCT. ${ }^{29}$ demonstrated that IA is safer, with better cosmetic appearance, technically more feasible, leads to faster recovery of intestinal function, is less invasive compared to EA. In the last 5years, the OrVil device, a common method of reconstruction in laparoscopy, has been showing positive results. We believe that reconstruction using the OrVil device should be used in patients with tumors of the cardia or lower third of the esophagus, because anastomosis can be formed high in the mediastinum which increases the probability of negative proximal resection margin after gastrectomy.

\section{Conclusions}

The review of the published studies and analysis of our observations showed insignificant differences in such essential criteria as quality of life and number of postoperative complications between gastrectomy with stapling and manual reconstruction. Roux-en-Y reconstruction of the gastrointestinal tract with pouch formation is associated with decreased risk of dumping syndrome and higher quality of life for patients. Therefore, this approach remains the preferable type of operation, as well as intracorporeal anastomosis, which is less invasive, safer and more esthetically satisfying for patients. For patients with tumors of the cardia and lower third of the esophagus the OrVil device is preferable.

\section{Conflicts of interest}

The authors have no ethical conflicts to disclose. The authors have no conflicts of interest to declare.

\section{Acknowledgments}

In the Author Contributions section, a short statement detailing the contributions of each person named as an author should be included. If an author is removed from or added to the listed authors after submission, an explanation and a signed statement of agreement confirming the requested change are required from all the initially listed authors and from the author to be removed or added.

\section{References}

1. Ferlay J, Colombet M, Soerjomataram I, et al. Global and Regional Estimates of the Incidence and Mortality for 38 Cancers: GLOBOCAN 2018. Lyon: International Agency for Research on Cancer; 2018. 
2. Sharma D. Choice of digestive tract reconstructive procedure following total gastrectomy: A critical reappraisal. Indian J Surg. 2004;66(5):270 276.

3. Conner PS. Report of a case complete resection of the stomach. New York, M News. 1884;45:578.

4. Schlatter C. A unique case of complete removal of the stomach: successful oesophagoenterostomy, Recovery. M Rec. 1897;52:909-1879

5. Japanese Gastric Cancer Association. Japanese gastric cancer treatment guidelines 2014 (ver. 4). Gastric Cancer. 2017;20(1):1-19.

6. Henley FA. Gastrectomy with replacement. Ann $R$ Coll Surg Engl. 1953;13(3):141-160.

7. Nakane Y, Michiura T, Inoue K, et al. A randomized clinical trial of pouch reconstruction after total gastrectomy for can- cer: Which is the better technique. Roux-en-Y or interposition? Hepatogastroenterology. 2001;48(39):903-907.

8. Adachi S, Inagawa S, Enomoto T, et al. Subjective and functional results after total gastrectomy; prospective study for long-term comparison of reconstructive procedures. Gastric Cancer. 2003;6(1):24-29.

9. Zhang JZ, Lu HS, Wu XY, et al. Influence of different procedures of alimentary tract reconstruction after total gas- trectomy for gastric cancer on the nutrition and metabolism of patients: a prospective clinical study. Zhonghua Yi Xue Za Zhi. 2003;83(17):1475-1478.

10. Iwahashi M, Nakamori M, Nakamura M, et al. Evaluation of double tract reconstruction after total gastrectomy in patients with gastric cancer: prospective randomized controlled trial. World J Surg. 2009;33(9):18821888.

11. Fuchs KH, Theide A, Englemann R, et al. Reconstruction of the food passage after total gastrectomy: randomized trial. World J Surg. 1995;19(5):698-706.

12. Yang YS, Chen LQ, Yan XX, et al. Preservation versus Non-preservation of the Duodenal Passage Following Total Gastrectomy: A Systematic Review. J Gastrointest Surg. 2013;17(5):877-886.

13. Kajitani K, Sato J. Evaluation of the procedures of total gastrectomy and proximal gastrectomy (in Japanese). J Jpn Surg Soc. 1965;66:1285-1287.

14. Resanovic A, Randjelovic T, Resanovic V, et al. Roux-en-Y Reconstruction in the treatment of Gastric Cancer. Pak J Med Sci. 2018;34(3):643-648.

15. Orr TG. A modified technic for total gastrectomy. Arch Surg. 1947;54(3):279-286.

16. Hunt CJ. Construction of food pouch from segment of jejunum as substitute for stomach in total gastrectomy. Arch Surg. 1952;64(5):601608 .

17. Herfarth $\mathrm{CH}$. Small intestine pouches as a therapeutic principle in stomach and large intestine replacement. Z Gastroenterol. 1988;26(8):397-403.

18. Zhang JZ, Lu HS, Wu XY, et al. Influence of different procedures of alimentary tract reconstruction after total gas- trectomy for gastric cancer on the nutrition and metabolism of patients: a prospective clinical study. Zhonghua Yi Xue Za Zhi. 2003;83(17):1475-1478.

19. Fein M, Fuchs KH, Thalheimer A, et al. Long-term benefits of Rouxen-Y pouch reconstruction after total gastrectomy: a randomized trial. Ann Surg. 2008;247(5):759-765.

20. Gertler R, Rosenberg R, Feith M, et al. Pouch vs. no pouch following tota gastrectomy: meta-analysis and systematic review. Am J Gastroenterol. 2009;104(11):2838.

21. Ito Y, Yoshikawa T, Fujiwara M, et al. Quality of life and nutritiona consequences after aboral pouch reconstruction following total gastrectomy for gastric cancer: randomized controlled trial CCG1101. Gastric Cancer. 2015;19(3):977-985.
22. Chen W, Jiang X, Huang H, et al. Jejunal pouch reconstruction after total gastrectomy is associated with better short- term absorption capacity and quality of life in early-stage gastric cancer patients, Chen et al. BMC Surgery. 2018;18(1):63.

23. Zonča $\mathrm{P}$, Malý T, Ihnát $\mathrm{P}$, et al. J-pouch versus Roux-en-Y reconstruction after gastrectomy: functional assessment and quality of life (randomized trial). Onco Targets and Therapy. 2017;10:13-19.

24. Dong H, Huang Y, Ding X, et al. Pouch size influences clinical outcome of pouch construction after total gastrectomy: A meta-analysis. World $J$ Gastroenterol. 2014;20(29):10166-10173.

25. Azagra JS, Goergen M, Ansay J, et al. Laparoscopic gastric reduction surgery. Preliminary results of a randomized, prospective trial of laparoscopic vs open vertical banded gastroplasty. Surg Endosc. 1999;13(6):555-558.

26. Ibanez FJA, Azagra JS, Erro Azcarate ML, et al. Laparoscopic gastrectomy for gastric adenocarcinoma. Long-term results. Rev Esp Enferm Dig. 2006;98(7):491-500.

27. Zheng XY, Pan Y, Chen K, et al. Comparison of Intracorporeal and Extracorporeal Esophagojejunostomy after Laparoscopic Total Gastrectomy for Gastric Cancer: A Meta-Analysis Based on Short-Term Outcomes. Chin Med J. 2018;131(6):713-720.

28. Woo J, Lee JH, Shim KN, et al. Does the difference of invasiveness between totally laparoscopic distal gastrectomy and laparoscopy-assisted distal gastrectomy lead to a difference in early surgical outcomes? A Prospective randomized trial. Ann Surg Oncol. 2015;22(6):1836-1843.

29. Zhang YX, Wu YJ, Lu GW, Xia MM. Systematic review and metaanalysis of totally laparoscopic versus laparoscopic assisted distal gastrectomy for gastric cancer. World J Surg Oncol. 2015;13:116.

30. Otsuka R, Hayashi H, Hanari N, et al. Laparoscopic double-trac reconstruction after total gastrectomy for postoperative duodenal surveillance: Case series. Ann Med Surg. 2017;21:105-108.

31. Kanaya S, Gomi T, Momoi H, et al. Delta-shaped anastomosis in totally laparoscopic Billroth I gastrectomy: new tech- nique of intraabdominal gastroduodenostomy. J Am Coll Surg. 2002;195(2):284e287.

32. $\mathrm{Hu}$ Y, Huang $\mathrm{C}$, Sun $\mathrm{Y}$, et al. Morbidity and mortality of laparoscopic versus open D2 distal gastrectomy for advanced gastric cancer: a randomized controlled trial. J Clin Oncol. 2016;34(12):1350-1357.

33. Zuiki T, Hosoya Y, Kaneda Y, et al. Stenosis after use of the doublestapling technique for reconstruction after laparoscopy-assisted total gastrectomy. Surgical Endoscopy. 2013;27(10):3683-3689.

34. Fukagawa T, Gotoda T, Oda I, et al. Stenosis of esophagojejuno anastomosis after gastric surgery. World J Surg. 2010;34(8):1859-1863.

35. Jeong O, Ryu SY, Zhao XF, et al. Short-term surgical outcomes and operative risks of laparoscopic total gastrectomy (LTG) for gastric carcinoma: experience at a large-volume center. Surg Endosc. 2012;26(12):3418-3425.

36. Zuiki T, Hosoya Y, Kaneda Y, et al. Stenosis after use of the doublestapling technique for reconstruction after laparoscopy-assisted total gastrectomy. Surg Endosc. 2013;27(10):3683-3689.

37. Duan W, Liu K, Fu X, et al. Semi-end-to-end esophagojejunostomy after laparoscopy-assisted total gastrectomy better reduces stricture and leakage than the conventional end-to-side procedure: A retrospective study. J Surg Oncol. 2017;116(2):177-183.

38. Wada N, Kurokawa Y, Takiguchi S, et al. Feasibility of laparoscopyassisted total gastrectomy in patients with clinical stage I gastric cancer. Gastric Cancer. 2014;17(1):137-140.

39. Zuiki T, Hosoya Y, Kaneda Y, et al. Stenosis after use of the doublestapling technique for reconstruction after laparoscopy-assisted total gastrectomy. Surg Endosc. 2013;27(10):3683-3689. 
40. Abdelmonim E, Gary A, Yvonne D, et al. Extending the reach of stapled anastomosis with a prepared OrVil ${ }^{\mathrm{TM}}$ device in laparoscopic oesophageal and gastric cancer surgery. Surg Endosc. 2015;29(4):961-971.
41. Ito $\mathrm{H}$, Inoue $\mathrm{H}$, Odaka $\mathrm{N}$, et al. Evaluation of the safety and efficacy of esophagojejunostomy after totally laparoscopic total gastrectomy using a trans-orally inserted anvil: a single-center comparative study. Surg Endosc. 2014;28(6):1929-1935. 